\title{
Methodological approaches to environmental assessment of rural settlements for justification of the sustainable development strategy
}

\author{
L.A. Mezhova ${ }^{1, *}$, E.A. Mikheeva ${ }^{1}, Y u . N$. Gladkiy $^{2}$, S.S. Popov ${ }^{3}$, and $O . Y u$. Sushkova ${ }^{4}$ \\ ${ }^{1}$ FSBEI of HE Voronezh State Pedagogical University, Voronezh, Russia \\ ${ }^{2}$ Herzen State Pedagogical University of Russia, Saint Petersburg, Russia \\ ${ }^{3}$ Military Educational and Scientific Center of the Air Force "Air Force Academy named after \\ Professor Zhukovsky N.Ye. and Gagarin Yu.A.", Voronezh \\ ${ }^{4}$ FSBEI of HE Voronezh State University, Voronezh, Russia
}

\begin{abstract}
The article studies methodological approaches to the environmental assessment of the quality of the environment and the quality of the population of rural settlements. The components of the "rural environment" of life activity include the factors of life support of rural residents or are the resource potential that determine the viability and vitality of the rural environment. The polystructurality of the living environment forms a special functional regime within the boundaries of rural settlements and agricultural activities. At the present stage, the population of rural settlements is experiencing the following negative factors: an increase in morbidity, genetic disorders, mortality, complication of social relations, and economic decline. The life activity of a rural resident is closely interrelated with the natural environment, agricultural activities and has wide spatial boundaries, including various spectra: home, household, objects of agricultural activity. The result of the processing is the compilation of morphometric maps. The map is the basis for creating predictive models for the course of exogenous processes. A monitoring algorithm, which collects data according to the scheme of a typical random nest selection, is important for the environmental analysis of the living environment of rural residents. The sample range, which guarantees the survey of various features of rural settlements, is calculated according to the rules of the sampling method for 145 villages. Rural settlements within the Voronezh region with a population of more than 500 people were studied in terms of medical and demographic indicators.
\end{abstract}

\footnotetext{
*Corresponding author: lidiya09mezhova@yandex.ru
} 


\section{Introduction}

The problem of assessing the living environment of a modern person occupies a leading place in the modern world within the development of a strategy for the balanced development of the "nature-population-economic activity" geosystem. Particular attention should be paid to the living environment of a rural person, while more than $25.44 \%$ of the population of Russia live in rural settlements.

Rural settlements in Russia performed demographic and economic functions in the development of the country. Various changes took place in the history of rural settlements the principles of organizing agricultural activities changed, which made changes in the way of life of villages and the nature of a rural settlement. The beginning of the XXI century is marked by contradictions and crises in the development of rural settlements. Revealing changes in the qualitative and quantitative indicators of the living environment of rural settlements is especially relevant. The manifestation of depopulation processes has been noted since the end of the XX century, which is expressed in a decrease in the population and the number of settlements. The structure of rural settlements and their socio-ecological and economic conditions are changing in the regions [8].

The habitat of rural settlements is considered one of the pressing problems of the beginning of the XXI century. The rural environment has specific spatial properties and living conditions. The rural settlement environment is a spatial geosystem that includes the homestead, the rural settlement, and the surrounding areas of agricultural activity. The villagers are constantly in contact with the life-supporting factors of the environment. The rural environment is a complex, multi-structural formation, which is formed by the interaction of natural, economic, man-made, and social environments. Many environmentally significant life-giving properties are eco-factors.

\section{Materials and Methods}

Assessment of the quality of the rural dweller's habitat is important for the formation of their sustainable balanced development. To assess the habitat of rural settlements, it is necessary to create a database that includes the collection of digital information and group it into two modules: "properties of the environment" and "properties of the population". The "environmental properties" module includes data on environmental factors. The module "population properties" takes into account medical and demographic indicators as indicators of the state of the environment and lifestyle. Settlements with more than 500 inhabitants were selected as accounting units. Accounting objects were selected by a selective method and according to the scheme of a typical nested random sample. The sample range should take into account different types of rural settlement environments with different properties. The nested selection of information on settlements should ensure the representativeness of the data in combination with the multiphase and location of the settlement in various geomorphological conditions, as well as in relation to the transport infrastructure of the territory. 145 settlements according to 55 indicators of the environment and 5 indicators of the quality of the population were examined on the Voronezh region territory. The sources of information used are cartographic materials, remote zoning data, monitoring, information, and statistical services of the regional and district municipal level, and Rosstat.

A geosystem study of the rural human habitat at the local spatial level, on the scale of individual settlements, was carried out. The information base in the study is represented by two modules for the implementation of system ideas with a focus on the anthropocentric approach. In order to optimize research procedures and improve the systematic study of rural geosystems, mathematical and statistical methods and GIS technologies were tested. 
The results of automatic processing of primary information were transformed into a series of a highly informative, new type, environmental maps, which reflect the spatial variability of the state of the environment and the quality of villagers in integral numerical indicators. The complex structure of anthropo-environmental relations with an indication of the priority of the impact of factors of the economic and man-made environment is fixed by the cartographic method within the geospace.

\section{Results and Discussion}

The life activity of the villager is closely interconnected with the natural environment, agricultural activity and has wide spatial boundaries, as well as closely interconnected with the relief. Geomorphological studies are necessary to identify negative natural and anthropogenic factors. The result of the processing is the compilation of morphometric maps. The map can be the basis for creating predictive models of the course of various geomorphological processes. To identify erosion processes, it is necessary to calculate the correlations between the variable values of the geosystem parameters, their graphical processing, coupled consideration of the evolutionary changes in the internal relationships and states of the elements of geosystems that exist in the same type and extreme conditions, climatic, geological and tectonic processes. This will reveal the self-oscillating mode of functioning of regional erosion geosystems. The rhythmic change in the phases of dismemberment and flattening reveals fluctuations. The phase change is caused by negative feedback and by self-regulation processes. The conjugated analysis will make it possible to determine the sequence of evolutionary changes in the morphometric properties of the erosional mesorelief and the peculiarities of the relationships.

The ecological effect of the environment depends on their combined and multidirectional influence, which is mutually exclusive in some types of settlements. On the territory of the region, eco-situations are obvious, where the unfavorable environment potential of one environment reduces the favorable environmental conditions of another, as well as the unfavorable conditions of weakly influencing eco-components are summed up, and their combined impact on the quality of rural residents is manifested in low indicators of the quality of the population.

The "rural environment" is a complex multicomponent entity, its various environmentally significant factors can be described using a variety of numerical indicators. Determination of the degree of the information content of individual indicators, automation of solving problems on the classification of geosystems on a quantitative, component, integrated basis $[5,6]$.

The use of a multidimensional classification algorithm is effective for the implementation of classification constructions. The classification approach using the multidimensional classification algorithm allows identifying the spatial patterns of the rural environment, and the elements of the functional regime, which will provide information on the dynamics of the living environment of the population.

Environmental study of the rural environment and its zoning at the regional level can contribute to determining the feasibility of controlling impacts on it and choosing the most optimal ways to improve it [2].

For a comprehensive assessment of the rural living environment, it is necessary to determine negative changes in the basic life-supporting properties of the environment, but for the implementation:

- there are lack of specific values and factual material about the state of the environment and the system of human interactions with his environment;

- the socio-ecological-economic environment of the villager has not been determined at the regional level; 
- medical and statistical data on the assessment of the quality of the population are not correct.

To identify patterns in the manifestation of processes and phenomena in rural areas, it is important to create a bank of environmental information, which is in various organizations at the present stage, which causes difficulties in a comprehensive assessment of rural settlements. Indicators of homogeneous populations have been developed to characterize the habitat by 50 parameters, which are the basis for the creation of cartographic and statistical models. The information and analytical model "habitat quality" is presented in Figure 1 .

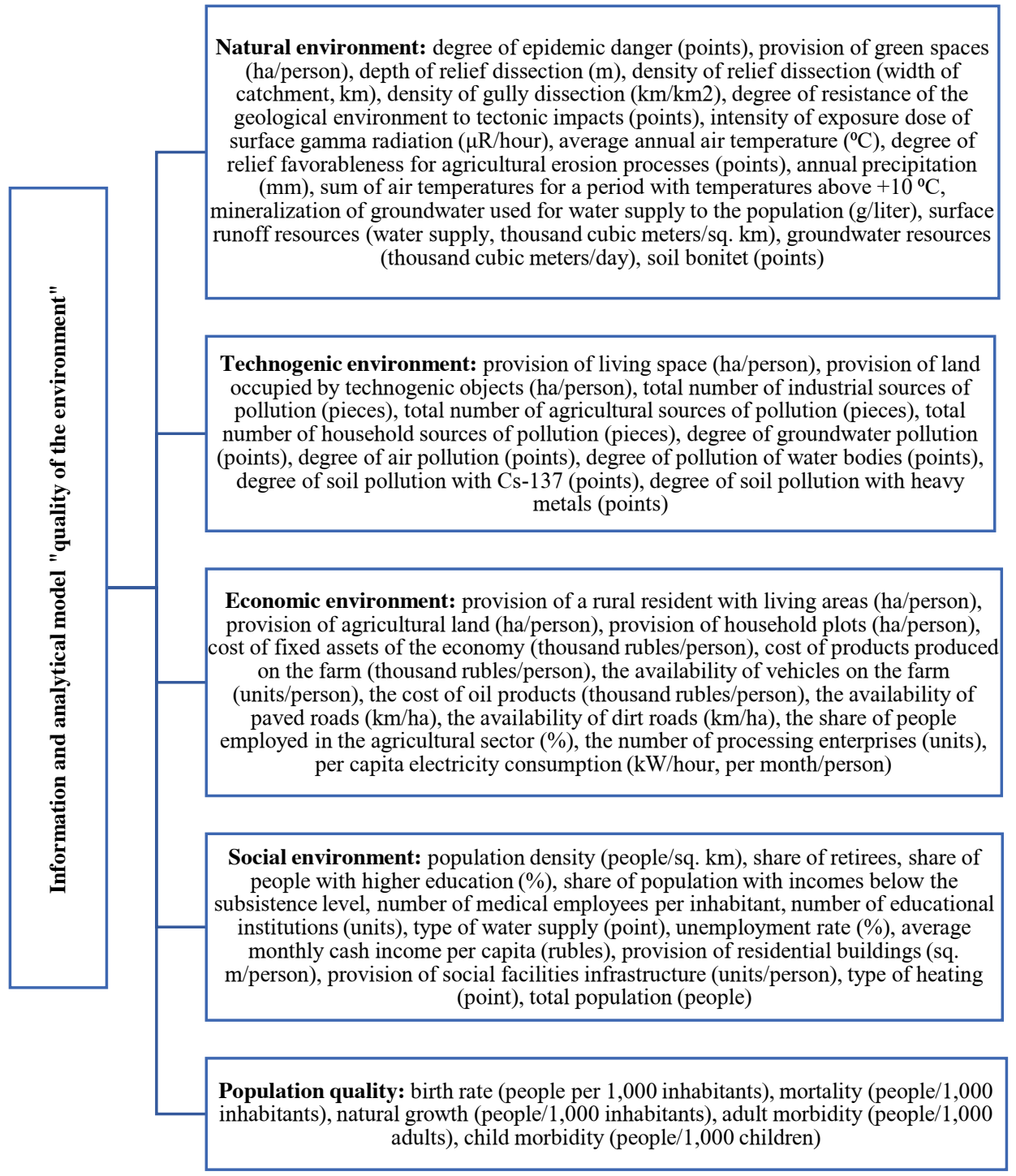

Fig. 1. Databases for information and analytical modeling "Habitat of a rural settlement"

The quality of the population of rural settlements is assessed on the basis of a binary division of the indicators of each settlement. As a result, 8 types of population quality were obtained, which differ from the average regional and all-Russian ones.

The procedure of the second algorithm consists in combining objects into classes 
according to the similarity of properties in the numerical values of all considered features. Formally, the classification problem is reduced to dividing an array of source data into classes in a multidimensional vector space. The classification algorithm identified 6 types of settlements, differing in a certain combination and mutual relationship of the entire set of indicators of the quality of the rural population [1].

Based on the classification results, cartographic modeling was carried out according to the estimated parameters of the quality of the population.

Cartographic modeling allowed us to prove the combined impact of all environmental factors, to measure the contribution of environmental factors and to identify spatial patterns of their manifestation [7]. The degree of influence of each factor is determined by correlation analysis. The initial set of population quality characteristics was described on the basis of factor analysis. Factor analysis established the integral influence of factors on the quality of the population, which made it possible to identify the direction of their influence on the territory of the region and municipal districts. Multidimensional classification makes it possible to assess the "quality of a rural settlement" and establish cause-and-effect relationships, which in the future will allow controlling the quality of the population. This approach makes it possible to group 5 classes according to the similarity of the joint quantitative manifestation of factors and causes.

To map the contribution of the environment to the quality of the population, it is necessary to summarize the data on the environment. The method of principal components is effective to study the factors. The results obtained by the component analysis establish the ecopotential of the environment within the boundaries of regional typological combinations on the integral map [3]. Conjugate analysis of all maps with a focus on the average long-term background values of the initial indicators on the quality of the population over the past 10 years in the region and in the country made it possible to give a qualitative assessment of the state of the environment and the quality of the population and to include these characteristics in the legends of maps that comprehensively represent ecosystems.

The compiled series of different types of ecological maps reveal the spatial patterns of the state of the environment of rural settlements, the quality of the population, and the specifics of environmental relations between them.

At the same time, it is possible to assess the response of the population to environmental impacts by the extent of its manifestation and display it on the map "Modern environmental situation of rural settlements" in gradations: dangerous, pathological, stressful.

There are two types of maps of environmental situations:

- the first - based on the results of the classification of the population according to the indicators of its quality;

- the second - based on the classification of indicators of "factor-causes" representing, in fact, the response of the population to the impact of the environment.

When comparing these options, incomplete correspondence is found in the differentiation of the geotopological space according to the characteristics of the environment, its effects, and the response of the population to these effects.

The reason for this discrepancy can be recorded on the maps that characterize the quality of the population of the studied region. The territorial structure of the rural population quality is clearly ordered: the most numerous types create clear bands of submeridial and sub-latitudinal orientation, sometimes interrupted by few groups. At the same time, the types in the bands are concentrated according to the principle "better - worse" and, moreover, the rhythmic alignment into bands is characterized by a tendency for a stepwise deterioration or improvement of indicators [4].

Medical and demographic indicators display information not only about the 
environmental burden, but also about the impact of internal mechanisms of selfdevelopment of rural residents. The generalized data of specially developed new environmental maps clearly and realistically identified the main environmental problems of rural areas within the boundaries of the Voronezh Region.

The use of effective methods of cartographic integration of quantitative information in the form of matrix legends enhances their applied value.

The compiled series of maps provides a data bank and a reference point for further solving the environmental problems of rural settlements.

Thus, the living environment of the rural population is determined in the following areas:

- assessment of negative changes in the viable and life-supporting properties of the environment;

- identification of the specifics of the environmental state of the environment and the relations of the rural population with the environment.

Mathematical and statistical methods of GIS technology have been tested in order to automate research procedures and improve the systematic study of rural settlements. The results of automatic processing of primary information are transformed into a series of ecological maps of a new type, which are highly informative and display the spatial variability of the state of the environment, the quality of the villagers, the environmental impact of the environment, and the response of the population in integral numerical indicators and in a complex (specially developed multidimensional matrix legends). The complex structure of anthropo-environmental relations with an indication of the priority of the impact of factors of the economic and man-made environment is fixed by the cartographic method within the geospace. The maps reflect problematic anthropoenvironmental situations of a high degree of complexity and extreme tension, which will justify priority directions in improving the living environment of the rural population.

Ecologically significant factors for rural residents are the natural water supply, territories, land agro-climatic conditions of the area, the availability of agricultural land to the population, and the quality of elements of the natural resource potential. The polystructure of the living environment forms its special functional regime within the boundaries of rural settlements and the relationship with agricultural activities. Currently, the population of rural settlements is experiencing a number of negative factors: an increase in morbidity, genetic disorders, mortality, a complication of social relations, economic problems, and pronounced depopulation processes. The developed methodological approach can be applied to solve problems of typological classification of objects. In terms of processing a large array of statistical information, which is later used for mapping the territory, this method has proven itself quite well. Analysis of the cartographic material reveals spatial patterns, and the changes under consideration reflect the state of the dynamics of geosystems.

\section{References}

1. Cătălina Ancuța, Martin Olaru, Nicolae Popa, Ramona Isfanescu-Ivan, Liviu JigoriaOprea, Carpathian journal of earth and environmental sciences, 10, 67 (2015)

2. E.V. Gorbenkova, E.V. Shcherbina, Vestnik MGSU, 10 (109), 1,107 (2017)

3. Indre Grazuleviciute-Vileniske, Vilma Karvelytė-Balbierienė, Journal of Architecture and Urbanism, 31, 213 (2007)

4. A.A. Kalininskaya, I.M. Son, S.I. Shlyafer, Problems of Social Hygiene, Public Health and History of Medicine, 2, 152 (2019)

5. S. Obraztsova, Eurasian Union Scientists, 7, 54 (2020) 
6. S.S. Popov, Astrakhan bulletin of environmental education, 4 (58), 117 (2020)

7. V.A. Rubtsov, N.K. Gabdrakhmanov, N.M. Biktimirov, M.R. Mustafin, R.R. Nurmiyeva, Springer Proceedings in Business and Economics (2020)

8. V.I. Frolov, EVR, 4 (2011), https://cyberleninka.ru/ 